\title{
Triangular Bézier Approximations to Constant Mean Curvature Surfaces
}

\author{
A. Arnal ${ }^{1}$, A. Lluch ${ }^{1}$, and J. Monterde ${ }^{2}$ \\ ${ }^{1}$ Dep. de Matemàtiques, Universitat Jaume I \\ Castelló, Spain \\ parnal@mat.uji.es, lluch@mat.uji.es \\ ${ }^{2}$ Dep. de Geometria i Topologia, Universitat de València, \\ Burjassot (València), Spain \\ monterde@uv.es
}

\begin{abstract}
We give a method to generate polynomial approximations to constant mean curvature surfaces with prescribed boundary. We address this problem by finding triangular Bézier extremals of the CMCfunctional among all polynomial surfaces with a prescribed boundary. Moreover, we analyze the $\mathcal{C}^{1}$ problem, we give a procedure to obtain solutions once the tangent planes for the boundary curves are also given.
\end{abstract}

\section{Introduction}

Surfaces with constant mean curvature (CMC-surfaces) are the mathematical abstraction of physical soap films and soap bubbles, and can be seen as the critical points of area for those variations that left the enclosed volume invariable. The study of these surfaces is actually relevant since there is a wide range of practical applications involving surface curvatures, ranging from rendering problems to real settings in automotive industry as measurement and calibration problems, for instance.

In general, the characterization of "area minimizing under volume constraint" is no longer true from a global point of view, since they could have self-intersections and extend to infinity. But locally, every small neighborhood of a point is still area minimizing while fixing the volume which is enclosed by the cone defined by the neighborhood's boundary and the origin.

An exhaustive discussion of the existence of surfaces of prescribed constant mean curvature spanning a Jordan curve in $\mathbb{R}^{3}$ can be found in $[2$. Given $H \in \mathbb{R}$ the functional $\mathcal{D}_{H}$ is defined as follows

$$
\begin{aligned}
\mathcal{D}_{H}(\vec{x}) & =\mathcal{D}(\vec{x})+2 H V(\vec{x}) \\
& =\frac{1}{2} \int_{\mathcal{T}}\left(\left\|\vec{x}_{u}\right\|^{2}+\left\|\vec{x}_{v}\right\|^{2}\right) d u d v+\frac{2 H}{3} \int_{\mathcal{T}}<\vec{x}_{u} \wedge \vec{x}_{v}, \vec{x}>d u d v,
\end{aligned}
$$

where $<,>$ and $\wedge$ denote the scalar and the cross product respectively.

If an isothermal patch is an extremal of the functional $\mathcal{D}_{H}$, then it is a CMCsurface. The "volume" term, $V(\vec{x})$, measures the algebraic volume enclosed in 
the cone segment consisting of all lines joining points $\vec{x}(u, v)$ on the surface with the origin. The first term, $\mathcal{D}(\vec{x})$, is the Dirichlet functional.

We will give a method to generate Bézier extremals of $\mathcal{D}_{H}$, for prescribed boundary curves and constant mean curvature. Our method lets to obtain approximations to CMC-surfaces, since we have considered the problem of minimizing this functional restricted to the space of polynomials.

Moreover, we will consider the $\mathcal{C}^{1}$ problem, that is, we give a way to generate a polynomial approximation to CMC-surface once the boundary curves and the tangent planes along them have been prescribed.

\section{Existence of Triangular Bézier Surfaces of Prescribed Constant Mean Curvature}

Here, we are not working with parametrizations, we are working instead with triangular control nets. So, our aim is to find the minimum of the real function $\mathcal{P} \rightarrow \mathcal{D}_{H}\left(\vec{x}_{\mathcal{P}}\right), \vec{x}_{\mathcal{P}}$ being the triangular Bézier patch associated to the control net $\mathcal{P}$.

The Dirichlet functional, $\mathcal{D}$, has a minimum in the Bézier case due to the following facts:

First, it can be considered as a continuous real function defined on $\mathbb{R}^{\frac{3(n-1)(n-2)}{2}}$, since there are $\frac{(n-1)(n-2)}{2}$ interior control points which belong to $\mathbb{R}^{3}$.

Second, the functional is bounded from below.

Third, the infima is attained: when looking for a minimum, we can restrict this function to a suitable compact subset.

On the other hand, the function assigning the value $V\left(\vec{x}_{\mathcal{P}}\right)$ to each control net, $\mathcal{P}$, with fixed boundary control points, has no global minimum. If that minimum existed, since spatial translations do not affect the curvature of the surface, we could suppose that the origin is located far enough away from the surface so that the control net is enclosed in a half-space passing through the origin. Let us move an interior control point, $P_{I_{0}}$, toward the origin. Then, a wellknown property of Bézier surfaces states that all the points of $\vec{x}(u, v)$ change in a parallel direction with intensity $B_{I_{0}}^{n}(u, v)$. Then, since the new cone segment is totally included in the initial one, its volume decreases.

As we said, the function, $\mathcal{P} \rightarrow \mathcal{D}\left(\vec{x}_{\mathcal{P}}\right)$, for control nets with fixed boundary always has a minimum and, as we have just seen, the function $\mathcal{P} \rightarrow V\left(\vec{x}_{\mathcal{P}}\right)$, never has a minimum. Therefore, by using the constant $H$ to balance both functions we can say that the function, $\mathcal{P} \rightarrow \mathcal{D}_{H}\left(\vec{x}_{\mathcal{P}}\right)$, will have a minimum only for $H \in[a,-a]$ for some constant $a \in \mathbb{R}$. It should be noted that when $H=0, \mathcal{D}_{H}$ is reduced to the Dirichlet functional, $\mathcal{D}$, and then there is a minimum, whereas when $\mathrm{H}$ is too big, the main term in $\mathcal{D}_{H}$ is $V$, and therefore the minimum does not exist.

The value of $a$ depends on the boundary control points and the symmetry of the interval, $[a,-a]$, is a consequence of the fact that reversing the orientation of a surface means a change in the sign of the mean curvature. A detailed explanation 
about the existence conditions of CMC-surfaces suited to a boundary and this dependency can be found in [2].

\section{The CMC-Functional Bézier Form}

The following proposition gives a characterization of an isothermal CMC-surface.

Proposition 1. [2] An isothermal patch, $\vec{x}$, is a CMC-surface if and only if

$$
\Delta \vec{x}=2 H \vec{x}_{u} \wedge \vec{x}_{v}
$$

Expression (10) is the Euler-Lagrange equation of the functional $\mathcal{D}_{H}$. Moreover, an isothermal patch satisfies the PDE in (1) if and only if it is an extremal of $\mathcal{D}_{H}$. In [1], it was proved that an extremal of the Dirichlet functional among all Bézier triangular surfaces with a prescribed boundary always exists and it is the solution of a linear system. Now we find two qualitative differences, the existence of the extremal of $\mathcal{D}_{H}$ can only be ensured with certainty when $|H| \leq a$, for a certain constant, $a$, depending on the boundary configuration, and they are computed as solutions of a quadratic system. Moreover, since the Euler-Lagrange equation of the functional $\mathcal{D}_{H}$, in Equation (1), is not linear we cannot determine a Bézier solution as a solution of a linear system of equations in terms of the control points.

Here we will give an expression of the CMC-functional in terms of the control points of a triangular Bézier surface, which implies that the restriction of the functional to the Bézier case can be seen as a function instead of as a functional.

The following two results will simplify the way to obtain the formula in terms of control points of the functional $\mathcal{D}_{H}$.

Proposition 2. The Dirichlet functional, $\mathcal{D}(\vec{x})$, of a triangular Bézier surface, $\vec{x}$, associated to the control net, $\mathcal{P}=\left\{P_{I}\right\}_{|I|=n}$, can be expressed in terms of the control points, $P_{I}=\left(x_{I}^{1}, x_{I}^{2}, x_{I}^{3}\right)$, with $|I|=\left|\left\{I^{1}, I^{2}, I^{3}\right\}\right|=n$ by the formula

$$
\mathcal{D}(\vec{x})=\frac{1}{2} \sum_{a=1}^{3} \sum_{\left|I_{0}\right|=n} \sum_{\left|I_{1}\right|=n} C_{I_{0} I_{1}} x_{I_{0}}^{a} x_{I_{1}}^{a}
$$

where

$$
C_{I_{0} I_{1}}=\frac{\left(\begin{array}{c}
n \\
I_{0}
\end{array}\right)\left(\begin{array}{c}
n \\
I_{1}
\end{array}\right)}{\left(\begin{array}{c}
2 n \\
I_{0}+I_{1}
\end{array}\right)}\left(a_{1}+a_{2}+2 a_{3}-b_{13}-b_{23}\right)
$$

and

$$
a_{r}=\left\{\begin{array}{ll}
0 \quad I^{r}=0, \\
\frac{I_{0}^{r} I^{r}}{\left(I_{0}^{r}+I^{r}\right)\left(I_{0}^{r}+I^{r}-1\right)} I^{r}>0
\end{array} \quad b_{r s}=\frac{I_{0}^{r} I^{s}+I_{0}^{s} I^{r}}{\left(I_{0}^{r}+I^{r}\right)\left(I_{0}^{s}+I^{s}\right)} .\right.
$$

Proof. The Dirichlet functional is a second-order functional, therefore we compute its second derivative in order to obtain the coefficients $C_{I_{0} I_{1}}$. 
The first derivative with respect to the coordinates of an interior control point $P_{I_{0}}=\left(x_{I_{0}}^{1}, x_{I_{0}}^{2}, x_{I_{0}}^{3}\right)$ where $I_{0}=\left(I_{0}^{1}, I_{0}^{2}, I_{0}^{3}\right)$ for any $a \in\{1,2,3\}$, and any $\left|I_{0}\right|=n$, with $I_{0}^{1}, I_{0}^{2}, I_{0}^{3} \neq 0$, is

$$
\frac{\partial \mathcal{D}(\vec{x})}{\partial x_{I_{0}}^{a}}=\int_{\mathcal{T}}\left(<\frac{\partial \overrightarrow{x_{u}}}{\partial x_{I_{0}}^{a}}, \overrightarrow{x_{u}}>+<\frac{\partial \overrightarrow{x_{v}}}{\partial x_{I_{0}}^{a}}, \overrightarrow{x_{v}}>\right) d u d v
$$

and the second derivative

$$
\begin{aligned}
\frac{\partial^{2} \mathcal{D}(\vec{x})}{\partial x_{I_{0}}^{a} \partial x_{I_{1}}^{a}} & =\int_{\mathcal{T}}\left(\left(B_{I_{0}}^{n}\right)_{u}\left(B_{I_{1}}^{n}\right)_{u}+\left(B_{I_{0}}^{n}\right)_{v}\left(B_{I_{1}}^{n}\right)_{v}\right)<e_{a}, e_{a}>d u d v \\
& =\frac{n^{2}}{2 n(2 n-1)} \frac{2 n(2 n-1)}{n^{2}} \frac{\left(\begin{array}{c}
n \\
I_{0}
\end{array}\right)\left(\begin{array}{c}
n \\
I_{1}
\end{array}\right)}{\left(\begin{array}{c}
2 n \\
I+I_{0}
\end{array}\right)}\left(a_{1}+a_{2}+2 a_{3}-b_{13}-b_{23}\right),
\end{aligned}
$$

where we took into account the formula for the product of the Bernstein polynomials and the value of its integral. Therefore

$$
C_{I_{0} I_{1}}=\frac{\left(\begin{array}{c}
n \\
I_{0}
\end{array}\right)\left(\begin{array}{c}
n \\
I_{1}
\end{array}\right)}{\left(\begin{array}{c}
2 n \\
I+I_{0}
\end{array}\right)}\left(a_{1}+a_{2}+2 a_{3}-b_{13}-b_{23}\right),
$$

where $a_{1}, a_{2}, a_{3}, b_{13}, b_{23}$ were defined in Equation (4).

Now, we will work the volume term of the CMC-functional.

Proposition 3. Let $\vec{x}$ be the triangular Bézier surface associated to the control net, $\mathcal{P}=\left\{P_{I}\right\}_{|I|=n}$, then the volume, $V(\vec{x})$, can be expressed in terms of the control points, $P_{I}=\left(x_{I}^{1}, x_{I}^{2}, x_{I}^{3}\right)$, with $|I|=n$, by the formula

$$
V(\vec{x})=\sum_{\left|I_{0}\right|=\left|I_{1}\right|=\left|I_{2}\right|=n} C_{I_{0} I_{1} I_{2}} x_{I_{0}}^{1} x_{I_{1}}^{2} x_{I_{2}}^{3}
$$

where

$$
C_{I_{0} I_{1} I_{2}}=\frac{\left(\begin{array}{c}
n \\
I_{0}
\end{array}\right)\left(\begin{array}{c}
n \\
I_{1}
\end{array}\right)\left(\begin{array}{c}
n \\
I_{2}
\end{array}\right)}{\left(\begin{array}{c}
3 n \\
I_{0}+I_{1}+I_{2}
\end{array}\right)}\left(d_{12}^{I_{0} I_{1} I_{2}}+d_{23}^{I_{0} I_{1} I_{2}}+d_{13}^{I_{0} I_{1} I_{2}}\right)
$$

with

$$
d_{r s}^{I J K}=\frac{I^{r} J^{s}-J^{r} I^{s}}{\left(I^{r}+J^{r}+K^{r}\right)\left(I^{s}+J^{s}+K^{s}\right)} .
$$

Proof. The term $V(\vec{x})$, is a cubical polynomial of the control points, so in order to compute the coefficients $C_{I_{0} I_{1} I_{2}}$ we will compute its third derivative.

The derivative with respect to a first coordinate $x_{I_{0}}^{1}$ of an arbitrary interior point $P_{I_{0}}=\left(x_{I_{0}}^{1}, x_{I_{0}}^{2}, x_{I_{0}}^{3}\right)$, where $\left|I_{0}\right|=n$ and $I_{0}^{1}, I_{0}^{2}, I_{0}^{3} \neq 0$, is given by 


$$
\begin{aligned}
& \frac{\partial V(\vec{x})}{\partial x_{I_{0}}^{1}}=\frac{1}{3} \int_{\mathcal{T}}\left(<\left(B_{I_{0}}^{n}\right)_{u} e^{1} \wedge \vec{x}_{v}, \vec{x}>+<\vec{x}_{u} \wedge\left(B_{I_{0}}^{n}\right)_{v} e^{1}, \vec{x}>\right. \\
& \left.+<\vec{x}_{u} \wedge \vec{x}_{v},\left(B_{I_{0}}^{n}\right) e^{1}>\right) d u d v \\
& =\int_{\mathcal{T}}<B_{I_{0}}^{n} e^{1} \wedge \vec{x}_{v}, \vec{x}>_{u}-<B_{I_{0}}^{n} e^{1} \wedge \vec{x}_{v u}, \vec{x}>+<\vec{x}_{u} \wedge B_{I_{0}}^{n} e^{1}, \vec{x}>_{v} \\
& \quad-<\vec{x}_{u v} \wedge B_{I_{0}}^{n} e^{1}, \vec{x}>+<\vec{x}_{u} \wedge \vec{x}_{v}, B_{I_{0}}^{n} e^{1}>d u d v .
\end{aligned}
$$

After computing the derivative with respect to an arbitrary first coordinate, we applied the integration by parts formula. Now, bearing in mind that

$$
\int_{\mathcal{T}}<B_{I_{0}}^{n} e^{1} \wedge \vec{x}_{v}, \vec{x}>_{u}=\int_{\mathcal{T}}<\vec{x}_{u} \wedge B_{I_{0}}^{n} e^{1}, \vec{x}>_{v}=0,
$$

since $B_{I_{0}}^{n}(1-v, v)=B_{I_{0}}^{n}(0, v)=B_{I_{0}}^{n}(u, 0)=B_{I_{0}}^{n}(u, 1-u)=0$ for $\left|I_{0}\right|=n$ with $I_{0}^{1}, I_{0}^{2}, I_{0}^{3} \neq 0$, and the properties of the cross and the scalar triple product, we obtain that

$$
\frac{\partial V(\vec{x})}{\partial x_{I_{0}}^{1}}=\frac{1}{3} \int_{\mathcal{T}}<\vec{x}_{u} \wedge \vec{x}_{v}, B_{I_{0}}^{n} e^{1}>.
$$

Now we must compute the derivative with respect to a second coordinate, $x_{I_{1}}^{2}$, of an arbitrary interior point, such that, as before, $\left|I_{1}\right|=n$ with $I_{1}^{1}, I_{1}^{2}, I_{1}^{3} \neq 0$.

Using the same process as before we have:

$$
\begin{aligned}
\frac{\partial^{2} V(\vec{x})}{\partial x_{I_{0}}^{1} \partial x_{I_{1}}^{2}} & =\frac{1}{3} \int_{\mathcal{T}}<\left(B_{I_{1}}^{n}\right)_{u} e^{2} \wedge \vec{x}_{v}, B_{I_{0}}^{n} e^{1}>+<\vec{x}_{u} \wedge\left(B_{I_{1}}^{n}\right)_{v} e^{2}, B_{I_{0}}^{n} e^{1}>d u d v \\
& =\int_{\mathcal{T}}\left(\left(B_{I_{0}}^{n}\right)_{u}\left(B_{I_{1}}^{n}\right)_{v}-\left(B_{I_{0}}^{n}\right)_{v}\left(B_{I_{1}}^{n}\right)_{u}\right)<e^{1} \wedge e^{2}, \vec{x}>d u d v .
\end{aligned}
$$

Finally we compute the derivative with respect to an arbitrary third coordinate $x_{I_{2}}^{3}$ with $\left|I_{2}\right|=n$ and such that $I_{2}^{1}, I_{2}^{2}, I_{2}^{3} \neq 0$, that is,

$$
\begin{aligned}
C_{x_{I_{0}}^{1} x_{I_{1}}^{2} x_{I_{2}}^{3}}=\frac{\partial^{2} V(\vec{x})}{\partial x_{I_{0}}^{1} \partial x_{I_{1}}^{2} \partial x_{I_{2}}^{3}}= & \int_{\mathcal{T}}\left(\left(B_{I_{0}}^{n}\right)_{u}\left(B_{I_{1}}^{n}\right)_{v}-\left(B_{I_{0}}^{n}\right)_{v}\left(B_{I_{1}}^{n}\right)_{u}\right) B_{I_{2}}^{n} d u d v \\
& =\frac{\left(\begin{array}{c}
n \\
I_{0}
\end{array}\right)\left(\begin{array}{c}
n \\
I_{1}
\end{array}\right)\left(\begin{array}{c}
n \\
I_{2}
\end{array}\right)}{\left(\begin{array}{c}
3 n \\
I_{0}+I_{1}+I_{2}
\end{array}\right)}\left(d_{12}^{I_{0} I_{1} I_{2}}+d_{23}^{I_{0} I_{1} I_{2}}+d_{13}^{I_{0} I_{1} I_{2}}\right)
\end{aligned}
$$

where we have achieved the last formula after computing the integral of the Bernstein polynomials and performing some simplifications like the following:

$$
\begin{aligned}
\int_{\mathcal{T}} B_{I_{0}-e_{1}}^{n-1} B_{I_{1}-e_{2}}^{n-1} B_{I_{2}}^{n} d u d v & =\int_{\mathcal{T}} \frac{\left(\begin{array}{c}
n-1 \\
I_{0}-e_{1}
\end{array}\right)\left(\begin{array}{c}
n-1 \\
I_{1}-e_{2}
\end{array}\right)\left(\begin{array}{c}
n \\
I_{2}
\end{array}\right)}{\left(\begin{array}{c}
3 n-2 \\
I_{0}+I_{1}+I_{2}-e_{1}-e_{2}
\end{array}\right)} B_{I_{0}+I_{1}+I_{2}-e_{1}-e_{2}}^{3 n-2} d u d v \\
& =\frac{\left(\begin{array}{c}
n \\
I_{0}
\end{array}\right)\left(\begin{array}{c}
n \\
I_{1}
\end{array}\right)\left(\begin{array}{c}
n \\
I_{2}
\end{array}\right)}{\left(\begin{array}{c}
3 n \\
I_{0}+I_{1}+I_{2}
\end{array}\right)} \frac{3 n(3 n-1)}{n^{2}} \frac{I_{0}^{1} I_{1}^{2}}{\left(I_{0}^{1}+I_{1}^{1}+I_{2}^{1}\right)\left(I_{0}^{2}+I_{1}^{2}+I_{2}^{2}\right)} .
\end{aligned}
$$


Lemma 1. The coefficients $C_{I J K}$ verify the following symmetry relations

$$
C_{I J K}=-C_{J I K}=C_{J K I}
$$

Proof. The symmetry of the coefficients $C$ 's is a direct consequence of the symmetry of d's: $d_{r s}^{I J K}=-d_{r s}^{J I K}$, which is immediate from its definition in Proposition 3 , since:

$$
d_{r s}^{J I K}=\frac{J^{r} I^{s}-I^{r} J^{s}}{\left(I^{r}+J^{r}+K^{r}\right)\left(I^{s}+J^{s}+K^{s}\right)} .
$$

In the following proposition we give a formula for the CMC-functional, $\mathcal{D}_{H}(\vec{x})$ in terms of the control net, $\mathcal{P}=\left\{P_{I}\right\}_{|I|=n}$, of the Bézier triangular surface, $\vec{x}$.

Proposition 4. Let $\vec{x}$ be the triangular Bézier surface associated to the control net, $\mathcal{P}=\left\{P_{I}\right\}_{|I|=n}$, where $P_{I}=\left(x_{I}^{1}, x_{I}^{2}, x_{I}^{3}\right)$ with $|I|=\left|\left\{I^{1}, I^{2}, I^{3}\right\}\right|=n$. The CMC-functional, $\mathcal{D}_{H}$, can be expressed by the formula

$$
\mathcal{D}_{H}(\vec{x})=\frac{1}{2} \sum_{a=1}^{3} \sum_{\left|I_{0}\right|=n} \sum_{\left|I_{1}\right|=n} C_{I_{0} I_{1}} x_{I_{0}}^{a} x_{I_{1}}^{a}+2 H \sum_{\left|I_{0}\right|=\left|I_{1}\right|=\left|I_{2}\right|=n} C_{I_{0} I_{1} I_{2}} x_{I_{0}}^{1} x_{I_{1}}^{2} x_{I_{2}}^{3}
$$

where

$$
C_{I_{0} I_{1}}=\frac{\left(\begin{array}{c}
n \\
I_{0}
\end{array}\right)\left(\begin{array}{c}
n \\
I_{1}
\end{array}\right)}{\left(\begin{array}{c}
2 n \\
I_{0}+I_{1}
\end{array}\right)}\left(a_{1}+a_{2}+2 a_{3}-b_{13}-b_{23}\right)
$$

with $a_{r}$ and $b_{r s}$ defined in Equation (4) and

$$
C_{I_{0} I_{1} I_{2}}=\frac{\left(\begin{array}{c}
n \\
I_{0}
\end{array}\right)\left(\begin{array}{c}
n \\
I_{1}
\end{array}\right)\left(\begin{array}{c}
n \\
I_{2}
\end{array}\right)}{\left(\begin{array}{c}
3 n \\
I_{0}+I_{1}+I_{2}
\end{array}\right)}\left(d_{12}^{I_{0} I_{1} I_{2}}+d_{23}^{I_{0} I_{1} I_{2}}+d_{13}^{I_{0} I_{1} I_{2}}\right)
$$

with $d_{r s}^{I J K}$ defined in Equation (6).

\section{Bézier Approximations to CMC-Surfaces}

We have just seen in Proposition 4 that the CMC-functional, is a function of the control points, so let us now compute its gradient with respect to the coordinates of an arbitrary control point. This will let us to give a characterization of the control net of the triangular Bézier extremals of $\mathcal{D}_{H}$, which are Bézier approximations to CMC-surfaces.

The gradient of the first addend, corresponding to the Dirichlet functional, with respect to the coordinates of a control point $P_{I_{0}}=\left(x_{I_{0}}^{1}, x_{I_{0}}^{2}, x_{I_{0}}^{3}\right)$

$$
\frac{\partial \mathcal{D}(\vec{x})}{\partial P_{I_{0}}}=\left(\sum_{|J|=n} C_{I_{0} J} x_{J}^{1} \sum_{|J|=n} C_{I_{0} J} x_{J}^{2}, \sum_{|J|=n} C_{I_{0} J} x_{J}^{3}\right)=\sum_{|J|=n} C_{I_{0} J} P_{J}
$$


So, let us consider the volume expression $V(\vec{x})=\sum_{|I|,|J|,|K|=n} C_{I J K} x_{I}^{1} x_{J}^{2} x_{K}^{3}$, and compute its gradient with respect to the coordinates of a control point $P_{I_{0}}$.

$$
\begin{aligned}
& \frac{\partial V(\vec{x})}{\partial P_{I_{0}}}=\sum_{|J|,|K|=n} C_{I_{0} J K}\left(x_{J}^{2} x_{K}^{3},-x_{J}^{1} x_{K}^{3}, x_{J}^{1} x_{K}^{2}\right) \\
& =\sum_{|J|,|K|=n} \frac{C_{I_{0} J K}-C_{I_{0} K J}}{2}\left(x_{J}^{2} x_{K}^{3},-x_{J}^{1} x_{K}^{3}, x_{J}^{1} x_{K}^{2}\right) \\
& =\frac{1}{2} \sum_{|J|,|K|=n} C_{I_{0} J K}\left(x_{J}^{2} x_{K}^{3}-x_{K}^{2} x_{J}^{3}, x_{K}^{1} x_{J}^{3}-x_{J}^{1} x_{K}^{3}, x_{J}^{1} x_{K}^{2}-x_{K}^{1} x_{J}^{2}\right) \\
& =\frac{1}{2} \sum_{|J|,|K|=n} C_{I_{0} J K} P_{J} \wedge P_{K} .
\end{aligned}
$$

Now we can characterize the triangular control net of an extremal of the CMCfunctional among all triangular Bézier patches constrained by a given boundary.

Proposition 5. A triangular control net, $\mathcal{P}=\left\{P_{I}\right\}_{|I|=n}$, is an extremal of the CMC-functional, $\mathcal{D}_{H}$, among all triangular control nets with a prescribed boundary if and only if:

$$
0=\sum_{|J|=n} C_{I_{0} J} P_{J}+H \sum_{|J|,|K|=n} C_{I_{0} J K} P_{J} \wedge P_{K}
$$

for all $\left|I_{0}=\left(I_{0}^{1}, I_{0}^{2}, I_{0}^{3}\right)\right|=n$ with $I_{0}^{1}, I_{0}^{2}, I_{0}^{3} \neq 0$, where the coefficients $C_{I_{0} J}$ and $C_{I_{0} J K}$ are defined in Equation (3) and Equation (5) respectively.

The last result lets us to obtain Bézier approximations to CMC-surfaces since we compute solutions to a restricted problem, that is, we find extremals of the functional $\mathcal{D}_{H}$ among all polynomial patches with prescribed border.

The following proposition characterizes the extremals of this restricted problem: $\vec{x}$ is an extremal of the functional $\mathcal{D}_{H}$ among all triangular Bézier patches with a prescribed boundary if and only if a weak version of the condition in Equation (1) is fulfilled.

Proposition 6. A triangular Bézier patch $\vec{x}$ is an extremal of the CMC-functional, $\mathcal{D}_{H}$, among all patches with a prescribed boundary if and only if:

$$
0=\int_{\mathcal{T}}\left(\Delta \vec{x}-2 H \vec{x}_{u} \wedge \vec{x}_{v}\right) B_{I_{0}}^{n} d u d v \quad \text { for all } \quad\left|I_{0}=\left(I_{0}^{1}, I_{0}^{2}, I_{0}^{3}\right)\right|=n
$$

with $I_{0}^{1}, I_{0}^{2}, I_{0}^{3} \neq 0$. 
Proof. We simply compute the gradient of the CMC-functional with respect to an arbitrary control point.

The boundary curves of our example in Fig. 1 describe an approximation to a circle. Therefore we obtain approximations to spheres. In Fig. 1 top, we have asked the interior control points to fulfill a symmetry condition:

$$
P_{112}=\left(a \cos \frac{4 \pi}{3}, a \sin \frac{4 \pi}{3}, b\right) \quad P_{121}=\left(a \cos \frac{2 \pi}{3}, a \sin \frac{2 \pi}{3}, b\right) \quad P_{211}=(a, 0, b)
$$

and we show three different approximations to CMC-surfaces.

The three surfaces at the bottom are obtained as a solution of the system of quadratic equations described in Equation (11). Here we don't ask for any kind of symmetry.
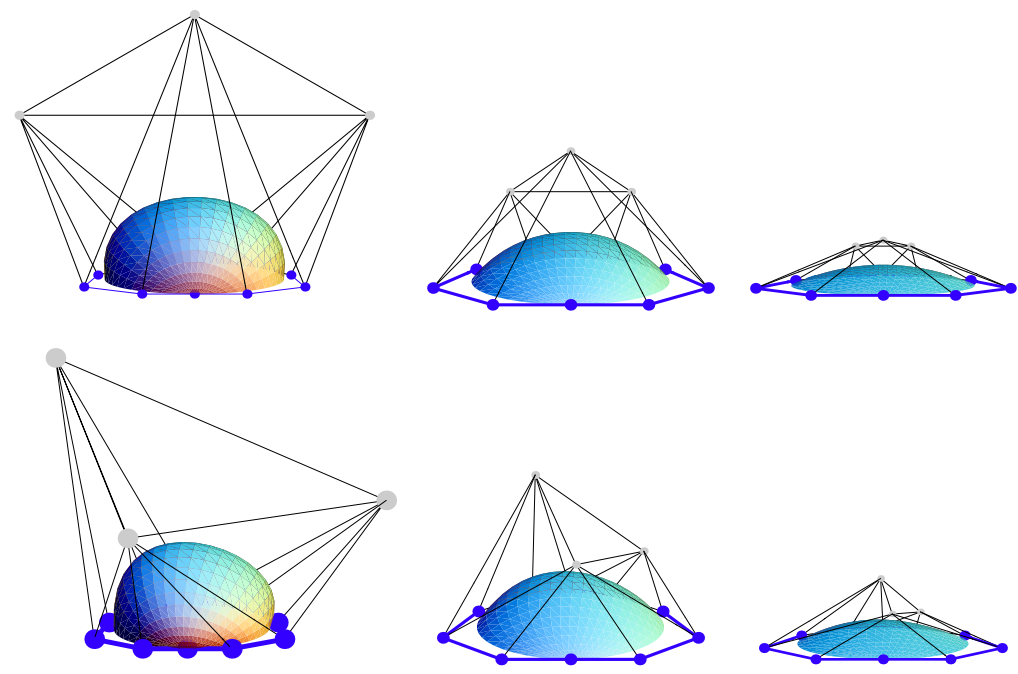

Fig. 1. These surfaces are approximations to CMC-surfaces with curvatures $H=-1.5$, $H=-1$ and $H=-0.5$ respectively

In Fig. 2 we present two more examples. The boundary curves in the first are built in such a way that any associated patch would be isothermal at the corner points and in the bottom surfaces in Fig. 2 the boundaries are approximations to three circular arcs, and therefore our results look like pieces of a sphere.

The resulting plots are pleasant and moreover they can be continuously deformed by the parameter $H$, thus allowing the designer to choose of the shape which best fits the objective. We maintain the good shapes we got with the Dirichlet results in [1], but now the choice of the curvature gives the designer another degree of freedom, although the surfaces are obtained as a solution of a quadratic system of the control points. 

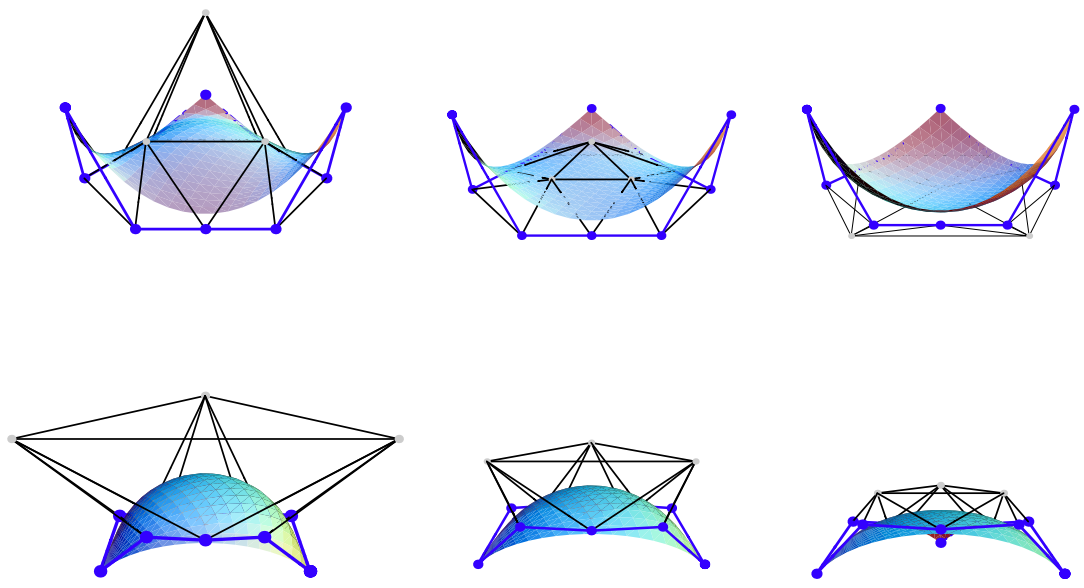

Fig. 2. These surfaces are approximations to CMC-surfaces with curvatures $H=-1$, $H=0$ and $H=1$ at the top and $H=-2, H=-1.5$ and $H=-1$ respectively at the bottom

\section{The $\mathcal{C}^{1}$ Problem}

In this section we will consider the prescription of not only the boundary but also the tangent planes along the boundary curves, the $\mathcal{C}^{1}$ problem. Now, the boundary and the next to the boundary control points are fixed, but again the extremals of the CMC-functional, where the other interior control points are considered as variables, can be computed.

Here we show an example. We prescribe the border control points along a planar equilateral triangle and three more lines of control points as it is shown in Fig. 3 .

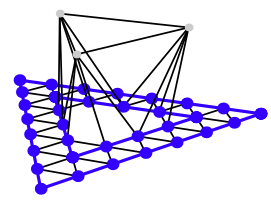

Fig. 3. The border control points and their neighboring lines of control points are prescribed

The following figures show approximations to CMC-surfaces obtained as a solution of the quadratic system of the control points in Equation (11), but now for all $\left|I_{0}=\left(I_{0}^{1}, I_{0}^{2}, I_{0}^{3}\right)\right|=n$ with $I_{0}^{1}, I_{0}^{2}, I_{0}^{3}>1$. The free points are the interior control points outside the boundary and its next line of control points. 

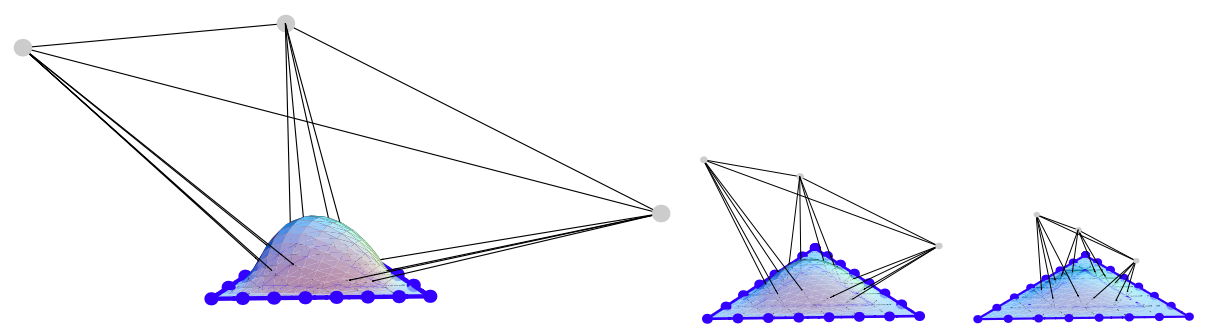

Fig. 4. These surfaces are approximations to CMC-surfaces with curvatures $H=-2$, $H=-1.5$ and $H=-1$ respectively

\section{Conclusions}

An isothermal patch has constant mean curvature $H$ if and only if it is an extremal of the functional

$$
\mathcal{D}_{H}(\vec{x})=\mathcal{D}(\vec{x})+2 H V(\vec{x}) .
$$

We have generated approximations to CMC-surfaces, since we have considered the problem of minimizing this functional restricted to the space of polynomials. We have obtained an expression of $\mathcal{D}_{H}$ in terms of the control points of a triangular Bézier surface. After that, we deduced the condition that a triangular control net must fulfill in order to be an extremal of $\mathcal{D}_{H}$ among all Bézier triangles with a prescribed boundary. This characterization of the Bézier extremals of $\mathcal{D}_{H}$ allowed us to compute them as a solution of a quadratic system of the control points. The surfaces that are obtained have regular shapes and have the advantage of allowing prescription of the desired curvature in addition to the boundary. This makes it possible to ensure, for a given boundary, the existence of a family of polynomial approximations to CMC-surfaces with this boundary and curvatures within a particular interval. Therefore, the prescription of the curvature in this method can be seen as another degree of freedom in comparison with the Dirichlet surface generation method in [1].

Finally, in the last section, we consider the $\mathcal{C}^{1}$ problem, that is, once the boundary curves and the tangent planes along them have been prescribed we give a way to generate a polynomial approximation to CMC-surface associated to this initial information.

\section{References}

1. Arnal, A., Lluch, A., Monterde, J.: Triangular Bézier Surfaces of Minimal Area. In: Kumar, V., Gavrilova, M.L., Tan, C.J.K., L'Ecuyer, P. (eds.) ICCSA 2003. LNCS, vol. 2669, pp. 366-375. Springer, Heidelberg (2003)

2. Struwe, M.: Plateau's problem and the calculus of variations. Mathematical Notes. Princeton University Press, Princeton (1988) 